\title{
The Technology Vicinity: A Location Based View on Technology
}

\author{
Harm-Jan Steenhuis ${ }^{1}$, Erik J. de Bruijn ${ }^{2}$ \\ ${ }^{1}$ Department of Psychology, North Carolina State University, Box 7801, Raleigh, NC 27695, USA \\ ${ }^{2}$ Technology and Development Group, University of Twente, PO Box 217, 7500 AE Enschede, The Netherlands
}

\begin{abstract}
The issue of technology transfer has been viewed from many different perspectives. In this case the focus is on the process of (production) technology transfer. One of the difficulties in studying international technology transfer is the definition of technology. The many technology definitions that exist are either too 'loosely' formulated or they require thorough expert knowledge. This results in difficulties with measuring technology and comparing different studies meaningfully. This study on technology transfer used technology as a sensitizing concept. Four case studies were performed and during these case studies a definition of technology was developed and refined, to obtain a meaningful representation of the technology at hand. The model enables the researchers on the transfer of technology to measure what is transferred. The research also showed that a technology can not be viewed in isolation but organizational factors and environmental factors influence the productivity of a technology. Therefore the functioning of technology should be viewed as dependent on its location.
\end{abstract}

\section{INTRODUCTION}

A range of issues related to technology transfer has been discussed in the literature but only recently research has been mainly focussed on the process of technology transfer [4, 5]. The difficulties of this type of the research lie in defining technology. A range of technology definitions is available. Technology, when related to technology transfer, is generally described as being embodied in three components: software, hardware and humanware, see [8]. Others include management, see $[12,15]$. There are also authors who identify technology transfer with knowledge transfer and distinguish different knowledge types such as industry specific knowledge, firm specific knowledge and system specific knowledge [9, 16, 19]. Tsang [17] makes a different distinction; he divides know-how into tacit and non-tacit.

Although these definitions are all conceptually valid, practically it is challenging to measure technology with these definitions. An exploration of other research fields reveals that technology can be viewed differently. In the strategic management literature technology is seen as an instrument, management of the technology and technology investments should contribute to the value of the enterprise, see $[2,10]$. In the production management literature technology is classified by production process characteristics such as unit or mass production, see $[6,20]$. In the marketing literature technology is viewed in relationship with a technology life cycle. This gives insight on when and how to sell technology, see $[3,11]$.

These definitions provide conceptual insights but the actual measurement of a technology remains extremely difficult. Even detailed definitions often have severe drawbacks because they require expert knowledge or a broad understanding of technological issues who are connected with the terms used. For example to be able to use the definition of the Technology Atlas Team [15], the technology needs to be rated by a comparison with alternative technologies worldwide. The conclusion is that measuring a technology remains difficult.

In the research this problem was handled by using technology as a sensitizing concept $[7$, p. 53], starting with the notion of four components: humanware, inforware, technoware and orgaware. As a result of the research, an improved model for technology measurement was developed and it was demonstrated that technology functioning is dependent on the environment [14].

\section{CASE STUDIES}

Four technology transfer case studies were carried out during which a model for measuring technology has been developed.

\section{A. Case study 1}

This involved the transfer of an aircraft manufacturing line from the UK to Romania. The transfer started in 1978 and was terminated in 1990. One of the main reasons for abandoning the project turned out to be the 'magnitude' of the technology. The technology, an entire aircraft manufacturing line, was large and it was (too) complex to transfer. It is possible to have a measurement of the magnitude of technology.

The case study analysis did not show the importance of an orgaware component or how such a component could be measured. Therefore the orgaware was omitted from the production technology definition. The humanware relates to the people who are handling the production technology. These people include operators but also managers. There are two important measurements concerning the humanware. First, to be able to produce with the technology it is necessary that the right amount of people is available. Second, these people should also have the required skills. There are basically two types of relevant skills. Technological skills include the ability to operate equipment and the ability to read (understand) and work from documentation. Management skills include the ability to plan, the ability to control and the ability to take corrective action. Skills can be increased by training and technical assistance. The use of the available skills is influenced by the attitude towards time and by the problem solving capability. The problem solving capability is related to the attitude towards decision making and the attitude towards responsibility.

Technoware consists of the factory buildings, including storage facilities, and manufacturing equipment. 
Manufacturing equipment covers two types of equipment: general manufacturing equipment and specific manufacturing equipment. General manufacturing equipment includes for example drills and rivet guns. Specific manufacturing equipment includes equipment specific for the product, for example jigs and platforms. The number of $\mathrm{m}^{2}$ and the number of manufacturing equipment used provides an indication of the size of the technoware.

Inforware consists of the amount of documentation (number of pages) needed for production. This documentation includes primarily product drawings, product support information and production process information but also the quality plan.

In aircraft manufacturing the combination of humanware, inforware and technoware has to be approved by the relevant aviation authorities. Not just any company is allowed to manufacture an aircraft. There are very high cost involved when it is only discovered in the end that the aircraft has defects. It is expensive to produce an aircraft and it is for safety reasons not possible to 'fix' things. This means that when it is discovered that the aircraft is not according to the standards, usually many parts have to be scrapped. Therefore in the entire industry an approach is chosen to have quality throughout the entire manufacturing process. This means for example that parts and materials can only be bought from approved sources. The second reason for approval issues is safety. In case an aircraft fails while it is in use the results can be catastrophic. Therefore an aircraft and the procedures to make that aircraft need to be approved by the aviation authorities. The company who produces the aircraft is required to work according to certain guidelines in order to 'guarantee' the product quality. The aircraft has to be built according to certain specifications and the entire organization has to be approved.

Apart from the three components of the technology it is also important to take inputs and outputs into consideration. The inputs are the materials and parts necessary in the production process, without these, production can not take place. The output is the product itself. The demand for this product can influence the production because if the demand is low it can result in a termination of the production. Demand can change due to various (external) reasons, for example changing noise regulations.

It was unambiguously observed that technology is dependent on the environment. Especially the differences in the environment of the source company and the destination company are important. Several differences between the environment of the aircraft production technology in the UK and the environment of the aircraft production technology in Romania existed, which made it difficult to transfer the aircraft manufacturing line. Examples of these were: in the political situation of Romania it was difficult for employees to obtain visas to travel to the UK for training. The infrastructure in Romania was less developed than in the UK, leading to long lead times for communication. The economic situation of Romania caused severe problems. Due to financial constraints, it was decided to use locally produced parts rather than purchase parts from the UK or other industrially advanced nations. The local industries in Romania were far less developed than in the UK. As a consequence, the lead-times for parts were longer than in the UK. In addition, the price of parts was often higher in Romania due to the smaller scale of production. Apart from this the UK company did not allow many of the materials because they didn't come from approved sources. Another observed difference was the organization structure. The Romanian company tried to copy the UK company's organization structure. Due to a different culture (the Romanian organization was used to centralization principles) this turned out to be unrealistic to achieve. At lower levels in the organization people were not accustomed to decision making or taking responsibility. This frequently led to rework and time delays. All the decisions for the entire project had to be made at the top level and often in co-operation with related government institutions.

\section{B. Case study 2}

This case involved the transfer of aircraft cockpit assembly activities from Canada to Romania. The transfer started in 1996. Presently continuous production is taking place in Romania. One of the technological variables that had a large influence on the transfer was the age of the technology. The transferred technology was a well-established technology. The case analysis showed that due to the age of the technology, much of the transferred information was outdated or incorrect.

The analysis showed that although management is an important factor, it should be separated from the humanware component. The operators are working with the technology or can be considered as part of the technology. Management however is 'guiding' the technology, it has a different impact. The importance of environmental differences was confirmed in this case and new insights were gained. As in the first case, the centralization culture in Romania led to problems. The under developed local industry in Romania led in this second case to the decision in the project to have parts supplied from Canada. The political instability in Romania resulted in a change of laws, which prompted customs to keep Canadian parts for an extended period of time. Only heavy interference of both Canadian and Romanian managers released the parts so that production could continue. Another factor was the difference in priority placed on the technology transfer project. The Canadian company didn't give a high priority to the project. A consequence of this attitude was that in many instances, when the destination company needed technical advice, it took a long time before they got the required answers.

\section{Case study 3}

The third case involved aircraft tail assembly. The assembly activities were transferred from the United Kingdom to Romania. The transfer started in 1997, while presently continuous production is taking place in Romania. The transferred technology was a new technology. A consequence of the newness was that much information was 
subject to changes. This phenomena had a large impact on the technology transfer.

The case analysis confirmed the earlier findings with respect to the technology components. Additional insight gained was that the combination of the technology components has specific characteristics.

Magnitude of technology refers to the total package of technology. It includes the amount of inforware (the number of drawings, the number of process planning sheets, the number of process specifications), the amount of humanware (number of man-hours necessary for production, number of training hours), and the amount of technoware (the necessary floor area, the necessary jigs and tools). It also includes the number of parts that have to be shipped (more parts lead to more logistic activities).

The first case involved a technology with a different magnitude than in the second and third cases, since it involved the transfer of a production technology for an entire aircraft whereas the others involved production technology for parts.

The type of production refers to production characteristics (mass, batch, unit). It is related to the division of humanware, inforware and technoware. Aircraft production technology is characterized as unit or small batch production. The relative amounts of humanware, inforware and technoware and their importance describe the type of production.

The age refers to how established the production technology is. A new technology is still in a state of flux. Many changes occur and this makes transferring such a technology difficult. The transfer of an old and wellestablished technology faces different challenges. An old technology has many tacit knowledge elements. Employees at the shopfloor level will use tools or will perform activities that are not mentioned in the drawings or process specifications. This is only discovered once technology is transferred to another organization, where this tacit knowledge does not exist.

The third case study confirmed the results of the first and second case study with regard to the importance of the technology environment. Poor working conditions, deficient infrastructure, different perceptions about the importance of time, decision making and responsibility taking, and different priorities placed on the project all influenced the smoothness of the transfer.

\section{Case study 4}

This case study involved the transfer of aircraft skin panel manufacturing from Germany to the Netherlands. It started in 1998 and presently continuous production is taking place in the Netherlands. Apart from confirmation of the previous case observations this case added insight into the importance of the environment. Although the industrial infrastructure of the Netherlands and Germany have many similarities, one of the main causes for a $120 \%$ budget overrun was that employees interpret things differently. Where the company in the Netherlands assumed that its processes were 'adequate', the German company assumed that this wasn't proven yet. Whereas none of the two parties were aware of this in advance, the processes of the Dutch manufacturer had to be approved, and this led to additional costs.

Even after the approval was given, there were several instances in which the drawings or process specifications were misinterpreted. Although it was generally assumed that drawings or process specifications could only be interpreted in one way, this is practically not so. Interpretation within the German company would not lead to problems, because everybody had a similar background (company training), but when information was transferred it caused problems in the Netherlands. Examples of this were the application of wrong rivets, drilling holes at the wrong locations and the manufacturing of aircraft skins that didn't fit. It was, in this last instance, only after extensive discussions between the companies that it became clear that the Dutch company had used a different method of interpreting the two-dimensional drawing of a three-dimensional part than the German company. As a result, the parts the Dutch company produced were not accepted.

\section{RESULTS}

From the case studies two important main findings can be derived. First, it is possible to define aircraft production technology in such a way that it is to a large extent possible to measure the magnitude of the technology. The development of the definition of technology was obtained through execution of these case studies. The result of this is a graphical representation of aircraft production technology as presented in Fig. 1.

It shows the production technology. The core of aircraft production technology consists of know-how (inforware) and means (humanware and technoware) for producing aircraft. Technoware contains the 'hardware' for producing the product and humanware the 'software' for producing the product. Inforware contains the documentation needed for production. The combination of these technology components has to be approved in this type of industry. Management is required in order to achieve that the technology is utilized productively.

The definition of aircraft production technology can not be viewed as an isolated matter. The studies made clear that a production technology can not be simply 'picked up and placed elsewhere'. There are three groups of factors influencing the technology transfer activities: technological factors, organizational factors, and environmental factors ${ }^{1}$. Fig. 2 depicts what has been defined as the Technology Vicinity, a representation of the important factors in the technology transfer process.

\footnotetext{
${ }^{1}$ It is important to realise that to determine this is difficult. Ideally, the factors that are listed should be independent. These factors should be the "solid rock of knowledge", determining the process outcomes but not influenced by anything else. The present level of knowledge is insufficient for getting to these independent factors (variables). Therefore a choice was made on which factors could be grouped conceptually and which factors were close to the sought independent variables.
} 


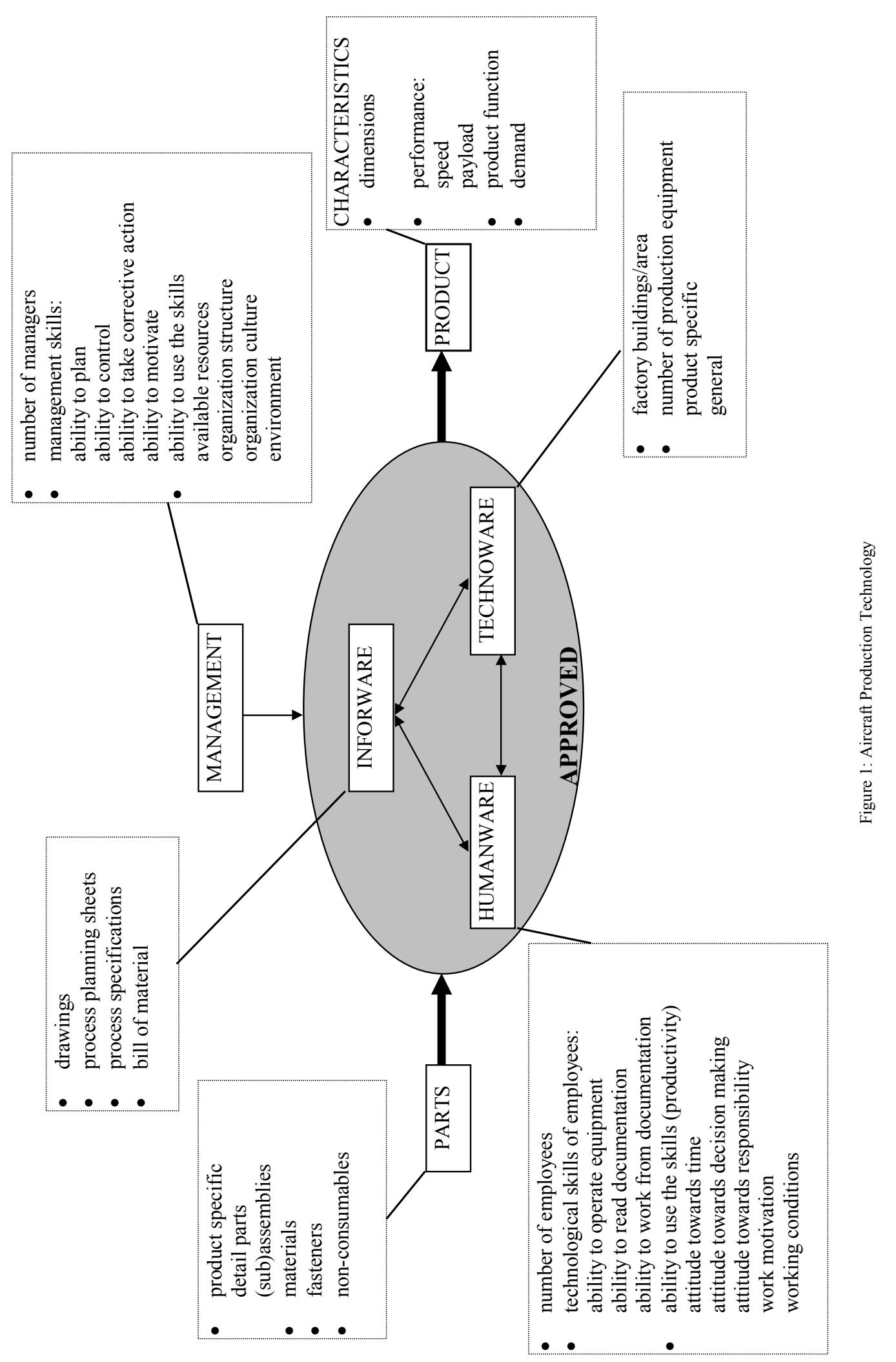




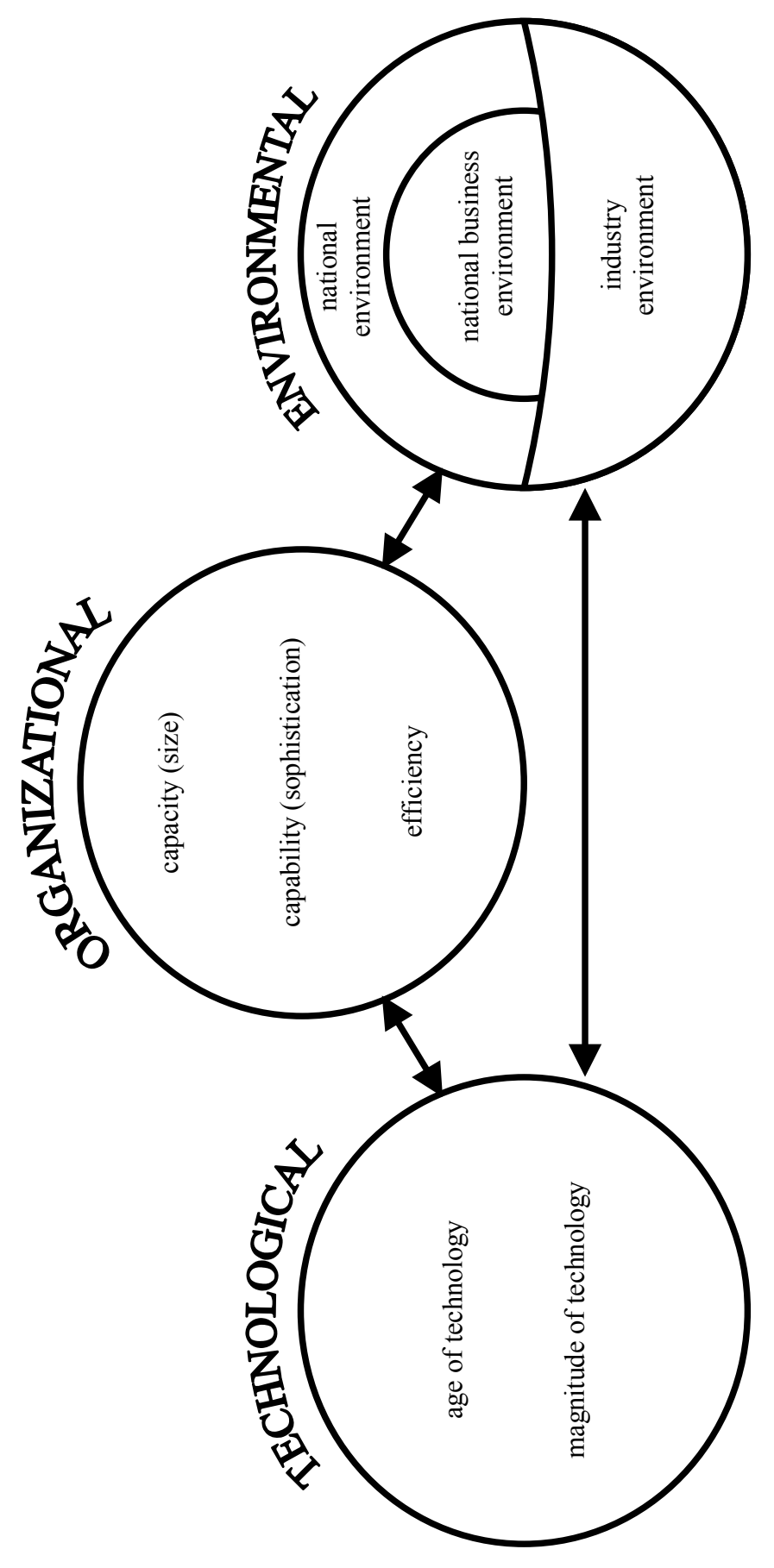

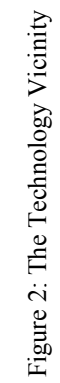




\section{A. Technological factors}

The technological factors are the magnitude of a technology and its age.

- The magnitude of a technology is determined by the different technological components as defined in the aircraft production technology definition (Fig. 1). A sizable technology is more difficult to transfer than a limited technology. In case a technology is very large, it is an option to transfer it in parts. The transfer of detail parts production is technologically more difficult than the transfer of assembly work. The transfer of assembly work is in general logistically more difficult (it is more difficult to keep track of all the detail parts) than the transfer of detail parts production.

- The age of a production technology is the elapsed time since first article production. If a technology is derived from an earlier type then it is older, because it relates back to the original technology. The age of a technology has several implications. First, the age of a technology is linked to the demand for the product. The aging of a technology can be a driving factor for a source company to sell it. Second, the age of a technology is related to its condition. Over time, a technology deteriorates. In particular, the condition of the technoware and inforware is important. An old technology will have older, worn out technoware that requires replacement. Old technology has a more stable inforware component. But, at the same time, the older a technology, the more shopfloor practice has occurred and consequently, the less 'up-to-date' or accurate the inforware is. Thus, when a technology is transferred, its condition plays a role.

\section{B. Organizational factors}

The organizational factors are the capacity of an organization, the capability of an organization, and the efficiency of an organization.

The capacity of an organization relates to the size:

- The number of machines and the capacity of each machine.

- The number of employees and the capacity of each individual.

The maximum capacity of an organization is not the same as the 'normal' capacity. For example, under pressure, an organization can require people to work overtime.

The capability of an organization relates to the level of sophistication of products it can deliver. This is determined by:

- The sophistication of the equipment.

- The skill level of the employees, which is related to their knowledge level. The knowledge level relates to an 'absolute' knowledge level and a 'relative' knowledge level. The 'absolute' knowledge level is the knowledge about manufacturing processes and procedures. The 'relative' knowledge level is the knowledge about the processes and procedures of other companies. This is important since it provides an indication of the likelihood of misinterpretations.

The capacity and the capability of an organization determine what can be produced and when. In case a technology is transferred from one organization to another, the destination company needs to have sufficient capacity and capabilities to be able to fabricate the product. If not, investments are needed to upgrade the existing capacity and capability.

The efficiency of an organization determines the amount of input an organization requires in order to achieve a certain output $^{2}$. The efficiency of an organization is determined by the following factors:

- The organizational structure: the manner in which tasks are sub-divided and the determination of the power to take decisions.

The organizational structure is important because it shows the division of work and the method of decision making. A different organizational structure leads to a different efficiency of an organization. Consequently, transferring a production technology to an organization with a different organizational structure will lead to a different efficiency.

- The management caliber is the extend of the knowledge and skills of the management. It is important because it indicates how well the organization is managed. In case the management caliber is low, the efficiency of the organization is likely to be negatively influenced.

- The organizational culture: delimiting acceptance of responsibility, motivation of the workforce, and attitude towards risk.

Indicators of culture that have been found in the case studies were acceptance of responsibility, motivation of employees, and attitude towards risk. If employees are not willing to accept responsibility, the efficiency tends to be low. Much time is wasted on formal decision making and allocating responsibilities. In case employees are not motivated, their attitude towards their work becomes lax which can lead to mistakes. The factor attitude towards risk is another factor is related to individuals in the organization (acceptance of responsibility) and to the overall organization. To limit the risk in the technology transfer, some source companies initially used a dual source strategy, i.e. they had two suppliers for the same part.

- The production methods: establishing the production philosophy, the difference in production lead time and the delivery interval, and the position on the learning curve also affect the efficiency of an organization.

\footnotetext{
2 The essence is productivity (real results/real sacrifice) or (actual output/actual input). This is a combination of effectiveness and efficiency [18]. Effectiveness is defined as (real results/norm results) and thus deals with the ability to reach an output. This is determined by the capacity and the capability. Both are already discussed, this leaves the efficiency. Efficiency is defined as (norm sacrifice/real sacrifice) and thus deals with how much input is necessary to reach an output.
} 
Differences in production philosophy lead to different input requirements, and consequently different efficiencies. The optimum use of resources for production is shown by an alignment of production (workstation) lead times and the delivery interval. When technology is transferred, a loss of efficiency is likely to occur due to the learning curve effects. The learning curve principle is widely used in the aircraft industry. The further along the learning curve, the better the efficiency of the organization (people will work faster and make less mistakes). If a technology is transferred from an organization that is already well along the learning curve (an established technology) then a loss in efficiency will occur (the destination company will be less efficient than the source company). The reason is that the destination company will start further back on the learning curve. In addition it should be noted that errors are human and will always occur. However, the rate of errors when a technology is newly transferred is likely to be higher than under normal circumstances. This is related to the knowledge at the destination company about the methods and procedures at the source company. In case there are different production philosophies, or if the knowledge about processes and methods used at the source company is relatively low at the destination company, there is a high probability that employees at the destination company will interpret the documentation incorrectly. These errors tend to diminish as the destination company becomes more familiar with the methods and processes of the source company. Training or technical assistance can also limit erroneous interpretations.

The availability of finances (liquidity) also influences the efficiency of an organization. If liquid assets are not available then the progress can be severely limited as was revealed in some of the case studies. The first case study demonstrated that a lack of finances could bring production to a complete stop because required inputs could not be purchased.

- The priority of the technology transfer to an organization is an important influencing factor on the efficiency of an organization. The case studies showed that the importance of the program to the source company is of particular importance for the efficiency of the destination company. All the transfers had many technical issues that needed to be resolved. In the case studies the source company placed a low emphasis on the project and, as a consequence, was negligent in solving technical problems. As a result, the production at the destination company was reduced.

- The location of an organization links the organization to its environment. It usually determines the language used (there might be a language barrier between a source company and a destination company). The distance between the source company and the destination company (time zones) also relates to the ease of communication between the two organizations.

\section{Environmental factors}

Three groups of environmental factors exist: national environmental factors, national business environmental factors, and industry environmental factors.

National environmental factors are:

- The political situation (international political position and the internal political stability) is a very important factor in a technology transfer. It affects the travel possibilities and the ease of providing technical assistance and training. A politically unstable situation represents a risk to the source company. For a technology sale, such as in the first case, risk is connected with payments. This risk can be decreased by the source company demanding bank guarantees from the destination company. In cases where a technology is shared with a supplier, as in the second, third and fourth case, risk is related to the ability of the destination company to meet the production schedule. It is difficult to hedge against this risk unless the source company uses a multiple source strategy.

- The economic situation concerns the type of economy (for example market economy or planned economy). It provides an insight on the ease of importing and exporting goods, and on the decision making power and the length of the decision making process. The condition of the economy is also a factor of the economic situation. It indicates the availability of financial means (especially relevant for state owned companies), and the ability to generate income, which reflects the productivity level of a country.

- The level of industrialization (the infrastructure, especially communication infrastructure and the level of education) has impact on the transfer of a production technology. The concept of the Technology Vicinity means that a production technology cannot simply be removed from the source company and placed at a destination company. The production technology is not independent of the organization and its environment. The level of industrialization is a variable in a technology transfer between a source company in an industrially developed country and a destination company in an industrially developing country. The level of industrialization has been established as an important factor in determining the production technology level at the source company. An underdeveloped infrastructure and a poor educational system in a destination country leads to problems with production. Furthermore, there is a high probability that the time (from ordering materials to finished product) for producing a part in an industrially developing country is longer than that required in an industrially developed country. - The national culture affects the time required to accomplish a specific task. An important variable found from the case studies was the attitude towards time. In countries where the national attitude towards time is relaxed, the efficiency will be low.

- The working conditions are a final important factor of the national environment. In poor working conditions, the employees will be less able to do a particular job. This results 
in mistakes and extended time required finishing the work. An example of this phenomena was the limited copying facilities in the Romanian factory, resulting in time wasted on walking to other buildings to get papers copied and waiting for it.

The national business environmental factors concern the level of related industries related to the level of industrialization. For a low level a choice can be made to either import materials and/or parts or to develop the local industries. Developing local industries requires time and is expensive. A low industrialization level increases the cost of materials and/or parts.

The industry environmental factors are:

- The level of concentration in the industry which gives an indication of the fierceness of competition between rival companies. The level of concentration in the aircraft industry is high. There is a very limited number of aircraft manufacturing companies worldwide.

- The strategic position of the organization in the industry is linked to the level of concentration in the industry. The strategic position in the industry influences whether or not a company can obtain more than average returns on investment. The strategic position also gives an indication of the bargaining power of the companies. The case studies showed that in the instances where the destination company became a supplier to the source company, the destination companies turned out to be in a weak bargaining position.

- The mandatory requirements in the industry (aircraft certification and production organization certification). Regardless of how well established a destination company is in its own environment, it must be certified to produce parts for a source company. In case the destination company has already produced for the source company, the process of certification might be relatively easy. Where co-operation has not occurred in the past, it often is a cumbersome process. The third and fourth case studies were examples that showed the complexity and importance of these certification procedures. Without certification, companies are taking the risk that the produced goods will not be allowed to be used. The certification of the aircraft itself is also important. If the aircraft is still being flight tested, the information tends to be unstable, complicating the technology transfer.

- The demand for aircraft is a further important factor. It is only (economically) sensible to produce an aircraft if there is a demand for it. Fluctuations in demand affect the production of aircraft and aircraft parts ${ }^{3}$. The demand for aircraft (parts) produced by a destination company can decrease in case the implementation of the technology transfer process takes too long and the technology may have become obsolete. This occurred in the first case. It was noted in the second, third, and fourth case studies that the source companies gave an

\footnotetext{
${ }^{3}$ Especially because inventory costs are high due to the high price of aircraft and aircraft parts.
}

indication of market demand. However, in all these cases the schedule had to be changed because of different actual demand characteristics. In addition to the real market demand, the switch from dual sources to a single source also affected the schedule. In the instances that this occurred in the case studies, the contractual schedules were based on a single source (the destination company) but for risk prevention the source company was initially also producing. When the switch was made to a single source, the parts made by the source company became available and thus the schedule at the destination company was postponed until these parts had been used.

\section{CONCLUSIONS}

When technology transfer is being studied it is imperative to have a good understanding of the technology that is being transferred. A literature review yielded numerous production technology definitions. Most of these definitions were general and difficult to operationalize in measurable factors. Some models were detailed but were difficult to apply due to required specific knowledge. In this research technology has been used as a sensitizing concept and an operational construct has been developed. The production technology model reflects the research focus on the time element of technology transfer, particularly with respect to the implementation of technology transfer. It enables measurement of the magnitude of a technology, rather than the complexity or sophistication. The model is a combination of process technology and product technology. An analytic division of technology in product technology and process technology is only meaningful when these two are also separated in practical situations. In the research both types of technology were transferred in all of the cases. This is a particular characteristic of the aircraft industry where, for safety reasons, process technology needs to be transferred. Product technology, notably drawings, is always transferred and therefore both types of technology are transferred in technology transfer projects in the aircraft industry.

The research analysis showed that the application of a production technology can not be isolated from the environment. The ease of transfer is determined by the differences in the environment of the source company and the destination company. The Technology Vicinity model provides a checklist of the most important environmental factors that have an impact on technology transfer. A technology has been shaped according to the source company's environment. It can therefore be expected that the same technology will be less productive at a destination company. The degree of productivity loss is determined by the combination of the factors in the Technology Vicinity model. Technology transferees should therefore examine these factors, especially the differences between source environment and destination environment, and assess the potential impact on a technology transfer project. 


\section{REFERENCES}

[1] N. Capon, R. Glazer, "Marketing and technology: a strategic coalignment," Journal of marketing, vol. 51, July 1987, pp. 1-14.

[2] T.J. Erickson, J.F. Magee, P.A. Roussel, K.N. Saad, "Managing technology as a business strategy," Sloan Management Review, Spring 1990, pp. 73-78.

[3] D. Ford, C. Ryan, "Taking technology to market," Harvard Business Review, March-April 1981, pp. 117-126.

[4] E.B. Grant, The international transfer of manufacturing: linking content and process, Ph.D. thesis, University of Cambridge, September 1997, unpublished.

[5] E.B. Grant, T.H.W. Minshall, M.J. Gregory, D.R. Probert, A.P. Steele, "Development of a manufacturing transfer process," Paper presented at the $14^{\text {th }}$ international conference on production research (ICPR), Osaka, Japan, 4-8 August 1997.

[6] T. Hill, Manufacturing strategy, The strategic management of the manufacturing function, Macmillan, Houndmills, 1985.

[7] J.M. Hutjes, J.A. van Buuren, De gevalsstudie: strategie van kwalitatief onderzoek, Boom, Meppel, 1992. In Dutch.

[8] W.J.J. Laseur, Managing technology transfer, Thieme, Zutphen, The Netherlands, 1991.

[9] E. Mansfield et al., Technology transfer, productivity, and economic policy, W.W. Norton \& Company, New York, 1982.

[10] J. Morone, "Strategic use of technology," California Management Review, Summer 1989, pp. 91-110.
[11] E.T. Popper, B.D. Buskirk, "Technology life cycles in industrial markets," Industrial Marketing Management, vol. 22, 1992, pp. 23-31.

[12] K. Ramanathan, "The polytrophic components of manufacturing technology," Technological forecasting and social change, vol. 46, 1994, pp. 221-258.

[13] L.W. Steele, Managing technology, McGraw-Hill, New York, 1989.

[14] H.J. Steenhuis, International technology transfer, Building theory from a multiple case-study in the aircraft industry, Ph.D. thesis, University of Twente, Enschede, the Netherlands, 2000. ISBN 903651469X.

[15] Technology Atlas Team, "Components of technology for resources transformation," Technological forecasting and social change, vol. 32, 1987, pp. 19-35.

[16] D.J. Teece, The multinational corporation and the resource cost of international technology transfer, Ballinger Publishing Company, Cambridge, 1976.

[17] E.W.K. Tsang, "Strategies for transferring technology to China", Long Range Planning, vol. 27, no. 3, 1994, pp. 98-107.

[18] J. in't Veld, Analyse van organisatie problemen, Een toepassing van denken in systemen en processen, Stenfert Kroese Uitgevers, Leiden, Antwerpen, 1992. In Dutch.

[19] H.W. Wallender III, Technology transfer and management in the developing countries: Company cases and policy analysis in Brazil, Kenya, Korea, Peru and Tanzania, Ballinger Publishing Company, Cambridge, 1979.

[20] J. Woodward, Industrial organization: theory and practice, Oxford University Press, London, 1965. 